Marseille; ${ }^{13}$ Department of Internal Medicine, CHU Grenoble, Grenoble: ${ }^{14}$ Department of Internal Medicine, Hôpital Archet 1, Nice, France

Background: Giant cell arteritis (GCA) is a large-vessel vasculitis affecting elderly people, and most frequently women (sex-ratio of 2.3). Some studies suggest an increased risk of malignancies in GCA.

Objectives: We aimed to describe the clinical, paraclinical characteristics and outcomes of GCA patients with concomitant malignancy and compare them to a control group without malignancy.

Methods: Patients with a diagnosis of GCA and of solid neoplasm or malignant blood disease, within one year before or after the diagnosis of vasculitis, were included. A random group of age-matched (3:1) control patients from our monocentric inception cohort of GCA patients from Caen University Hospital was constituted.

Results: Twenty-four observations were collected (median age 75.5 years). All fulfilled $\geq 3 / 5$ ACR criteria. Temporal artery biopsy was positive in 17 cases $(70.8 \%)$. There were 1 active $(4.2 \%)$ and 9 former (37.5\%) smokers. Only 1 patient had a previous prostate cancer. Malignancies were 10 malignant blood diseases (41.7\%, 3 chronic lymphoid leukemias, 3 essential thrombocythemias, 1 myeloma, 1 chronic myelomonocytic leukemia, 1 MALT lymphoma, 1 Waldenström's macroglobulinemia) and 14 solid neoplasms (58.3\%, 3 lung, 3 breast, 2 prostate, 1 thyroid, 1 colon, 1 pleural cancers, 1 melanoma, 1 Kaposi's sarcoma and 1 Merkel cell carcinoma). Malignancy was diagnosed at a median of 1 month after GCA diagnosis in 21 patients and before in the other 3 . Diagnosis of malignancy was made in consultation in 5 patients ( 3 skin cancers and 2 breast cancers), on lab tests in 13 (thrombocytosis, anemia or increased prostate specific antigen) and on imaging in 6 . Treatments of malignancy included chemotherapy alone in 8 patients (33.3\%), simple monitoring in 6 patients $(25 \%)$, surgery alone in 4 patients $(16.7 \%)$, surgery and radiotherapy and/or chemotherapy in 4 patients $(16.7 \%)$, decrease of corticosteroids in 1 patient, and 1 patient was lost to follow-up. Two patients $(8.3 \%)$ died from infectious complications, 8 patients $(33.3 \%)$ had a GCA relapse, including one with concomitant malignancy relapse. After a median follow-up of 16 months [0-134], 5 patients $(20.8 \%)$ were weaned from steroids, all considered in malignancy remission. Seven patients $(29.1 \%)$ were still under chemotherapy, 9 patients (37.5\%) were considered to be in malignancy remission. There were more males in patients with concomitant malignancy, compared to the control group (respectively 15/24 and 21/72, $p<0.005$ ).

Conclusions: Our study shows an over-representation of male gender in GCA with concomitant malignancy. Vasculitis outcomes were not influenced by the malignancy treatment. The diversity of malignancies encountered in this study raises the issue of an incidental association. Initial clinical and paraclinical followup dictated by vasculitis may have led to an early identification of associated malignancy, and thus represent a lead time bias.

Disclosure of Interest: None declared

DOI: 10.1136/annrheumdis-2017-eular.1291

\section{AB0589 THE ROLE OF ANCA SPECIFICITY IN THE CLINICAL MANIFESTATIONS AT DISEASE ONSET: COMPARISON BETWEEN PATIENTS WITH GRANULOMATOSIS WITH POLYANGIITIS AND MICROSCOPIC POLYANGIITIS}

S. Monti ${ }^{1}$, M. Felicetti ${ }^{2,3}$, S. Balduzzi ${ }^{1}$, R. Padoan ${ }^{2}$, A. Berti ${ }^{3}$, G. Paolazzi $^{3}$, G. Brunori ${ }^{3}$, F. Schiavon ${ }^{2}$, R. Caporali ${ }^{1} .{ }^{1}$ Rheumatology, University of Pavia, IRCCS Policlinico San Matteo Foundation, Pavia; ${ }^{2}$ Rheumatology, Department of Medicine DIMED, University of Padova, Padova: ${ }^{3}$ Rheumatology, Santa Chiara Hospital, Trento, Italy

Background: ANCA specificity, rather than clinical diagnosis, has been suggested to influence the phenotype and clinical course of ANCA associated vasculitis (AAV) $(1,2)$.

Objectives: To investigate differences in clinical presentation at disease onset between MPO-ANCA-positive granulomatosis with polyangiitis patients (MPOGPA), PR3-ANCA-positive-GPA (PR3-GPA), and MPO-ANCA-positive microscopic polyangiitis (MPO-MPA).

Methods: Clinical records of AAV patients from three third level rheumatologic centers in Northern Italy were retrospectively analyzed.

Results: Of the 133 AAV patients included, 84 were PR3-GPA, 24 MPO-GPA, and 25 MPO-MPA. Patients with MPO-MPA were significantly older at diagnosis compared to both PR3-GPA and MPO-GPA (average age $63 \pm 10,49 \pm 15,55 \pm 29$, respectively) (Table 1 ).

Patients with MPO-GPA experienced a significant diagnostic delay compared to PR3-GPA ( $17 \pm 30$ vs $7 \pm 14, p=0.02)$. ENT involvement was equally frequent in both GPA groups despite ANCA specificity, and significantly more represented than the MPO-MPA group $(68 \%, 71 \%$ and $17 \%$ respectively; $p<0.001)$. Figure 1 . Renal involvement was significantly more frequent in MPO-MPA patients (100\%) compared to GPA $(p<0.001)$, without differences between MPO-GPA $(46 \%)$ and PR3-GPA (65\%). Alveolar haemorrhage (DAH) was an onset manifestation mainly in MPO-MPA compared to the other two groups (24\% vs $7 \%$ in PR3GPA; $p=0.02$ ). Cutenous manifestations, mainly purpura, were significantly more reported in PR3-GPA compared to MPO-MPA (29\% vs 4\%; $p=0.03$ ).

Conclusions: Clinical phenotype of GPA at disease onset did not seem to be influenced by ANCA specificity. Despite ANCA positivity (PR3 or MPO), GPA patients were significantly different from MPA.
Table 1. Clinical characteristics of patients with GPA and MPA at disease onset according to ANCA specificity

\begin{tabular}{|c|c|c|c|c|}
\hline & $\begin{array}{c}\text { GPA-PR3 (A) } \\
(n=84)\end{array}$ & $\begin{array}{c}\text { GPA-MPO (B) } \\
(n=24)\end{array}$ & $\begin{array}{c}\text { MPA-MPO (C) } \\
(n=25)\end{array}$ & $\mathrm{p}$ \\
\hline Male/female n (\%) & $46(55 \%) / 38(45 \%)$ & $10(42 \%) / 14(58 \%)$ & $10(40 \%) / 15(60 \%)$ & 0.29 \\
\hline $\begin{array}{l}\text { Age at disease onset } \\
\text { (average } \pm S D)\end{array}$ & $49 \pm 15$ & $55 \pm 19$ & $63 \pm 10$ & $\begin{array}{c}\quad p<0.001 \\
\text { A vs } C p<0.001 \\
\text { B vs } C p=0.03\end{array}$ \\
\hline $\begin{array}{l}\text { Diagnostic delay } \\
\text { (months } \pm \text { SD) }\end{array}$ & $7 \pm 14$ & $17 \pm 30$ & $10 \pm 19$ & 0.07 \\
\hline Systemic symptoms & $54(65 \%)$ & $16(67 \%)$ & $19(76 \%)$ & 0.59 \\
\hline ENT & $55(68 \%)$ & $17(71 \%)$ & $4(17 \%)$ & $<0.001$ \\
\hline Pulmonary & 57 (69\%) & $16(67 \%)$ & $16(64 \%)$ & 0.86 \\
\hline Cutaneous & $24(29 \%)$ & $5(21 \%)$ & $1(4 \%)$ & 0.03 \\
\hline Ocular & $16(20 \%)$ & $3(12 \%)$ & $2(8 \%)$ & 0.34 \\
\hline Cardiovascular & $4(5 \%)$ & $2(9 \%)$ & $1(4 \%)$ & 0.77 \\
\hline Gastrointestinal & $2(3 \%)$ & 0 & 0 & 0.56 \\
\hline Renal & $53(65 \%)$ & $11(46 \%)$ & $25(100 \%)$ & $<0.001$ \\
\hline Nervous system & $26(31 \%)$ & $8(33 \%)$ & $8(35 \%)$ & 0.94 \\
\hline
\end{tabular}

Figure 1. Differences in onset clinical manifestations among PR3-GPA, MPO-GPA and MPO-MPA

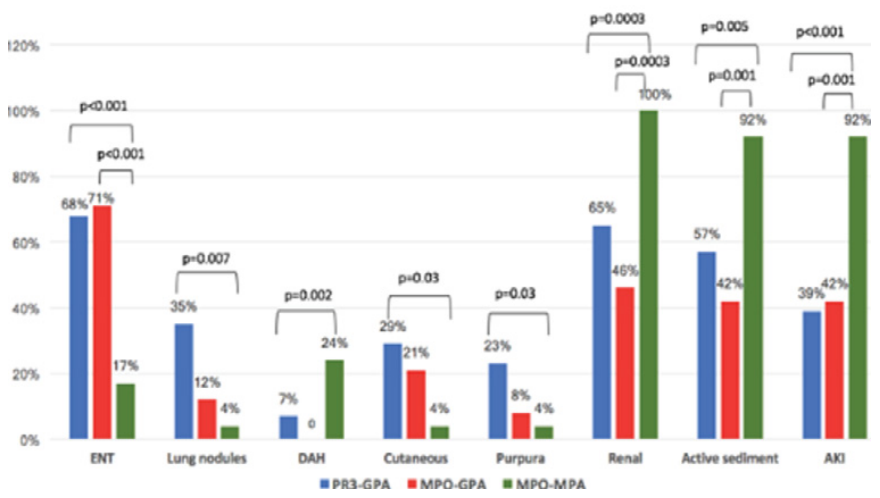

References:

[1] Schirmer JH, Wright MN, Herrmann K, et al. Myeloperoxidase-Antineutrophil Cytoplasmic Antibody (ANCA)-Positive Granulomatosis With Polyangiitis (Wegener's) Is a Clinically Distinct Subset of ANCA-Associated Vasculitis: A Retrospective Analysis of 315 Patients From a German Vasculitis Referral Center. Arthritis Rheumatol Hoboken NJ 2016;68:2953-63.

[2] Miloslavsky EM, Lu N, Unizony S, et al. Myeloperoxidase-Antineutrophil Cytoplasmic Antibody (ANCA)-Positive and ANCA-Negative Patients With Granulomatosis With Polyangiitis (Wegener's): Distinct Patient Subsets. Arthritis Rheumatol 2016;68:2945-2952.

Disclosure of Interest: None declared

DOI: 10.1136/annrheumdis-2017-eular.4760

\section{AB0590 PERFORMANCE OF 2017 ACR/EULAR PROVISIONAL CLASSIFICATION CRITERIA FOR GRANULOMATOSIS WITH POLYANGIITIS IN CHILEAN POPULATION}

S.F. Elgueta, L. Ricci Larrea, D. Vargas, P. Wurmann, I. Goecke. Hospital Clínico Universidad de Chile, Santiago, Chile

Background: Anca Associated Vasculitis (AAV), are a group of necrotizing primary vascultitis, whith multisistemic manifestation, of unknown etiology. The variants are: Microscopic Polyangiitis (MPA), Granulomatosis with polyangiitis (GPA), Granulomatosis whith Polyangiitis and Eosinophilia (GPE) and AAV limited to one organ. Until now, there are no diagnostic criteria for AAV. Therefore definitions, as Chapell Hill consensus Conference Nomenclature, classification criteria and the physician judgement are used for diagnosis. Currently the DCVAS (Diagnosis and Classification Criteria in Vasculitis) proyect is developing diagnostic criteria for AAV, using data-driven methods. The preliminary DCVAS classification criteria for granulomatosis whith polyangiitis has been recently realesead.

Objectives: To evaluate and compare the acuraccy of ACR/EULAR 2017 provisional Classification Criteria for GPA whith the ACR 1990 Classification Criteria In Chilean patients with AAV.

Methods: All adult patients ( $>18$ yo) with diagnoses of AAV according to their rheumatologist judgment, from 2000-2016 at the University of Chile, Clinical Hospital $(\mathrm{UCCH})$, were included. Clinical variables of interest were extracted form medical chart and AAV database, wich is kept for these patients at the Rheumatology Section of UCCH. Based on that data, the Classification criteria ACR 1990 and 2017preliminary ACR/EULAR (DCVAS) classification criteria for GPA were applied to each individual. Sensibility, especifity, Likehood ratio (LR $+/-$ ), predictive values (PPV/NPV) and accuracy were calculated for both sets of Criteria as compared to Clinical diagnosis

Results: 93 patient were included in the study. 59 patients with GPA, 33 with 
MPA and 1 with GPE (Patients characteristics are described in Table 1). From the 59 patients with GPA according to clinical judgment, 34 fullfilled the ACR 1990 criteria $(57,63 \%)$ whereas 51 (86,44\%) fullfilled the ACR/EULAR 2007 criteria. From the 33 patients diagnosed as having MPA $9(27,27 \%)$ and and $3(9.09 \%)$ fulfilled classification criteria for GPA according to ACR 1990 criteria and 2017 ACR/EULAR preliminary criteria, respectively. The patient with GPE did no classify as GPA by either set of criteria. The values for Sensibility, Espicifity, LR+ LR-, PPV and NPV in our population for both Criteria sets, are described in table 2.

Table 1. Patient characteristics

\begin{tabular}{|l|l|l|l|l|l|}
\hline & N & Gender (F) & $\begin{array}{l}\text { Age, Av } \\
\text { (S.D.) }\end{array}$ & $\begin{array}{l}\text { ACR } \\
1990+ \\
\text { for GPA }\end{array}$ & $\begin{array}{l}\text { ACR/ } \\
\text { EULAR } \\
2017+ \\
\text { for GPA }\end{array}$ \\
\hline GPA & $59(63.44 \%)$ & $30(50.85 \%)$ & $\begin{array}{l}46.54 \\
(13.32)\end{array}$ & $\begin{array}{l}34 \\
(57,63 \%)\end{array}$ & $\begin{array}{l}51 \\
(86,44 \%)\end{array}$ \\
\hline MPA & $33(35.48 \%)$ & $23(69.7 \%)$ & $\begin{array}{l}62,72 \\
(2.11)\end{array}$ & $\begin{array}{l}9 \\
(27,27 \%)\end{array}$ & $3(9,09 \%)$ \\
\hline GPE & $1(1.08 \%)$ & 1 & 62 & 0 & 0 \\
\hline Total & 93 & $39(41.94 \%)$ & $\begin{array}{l}52,45 \\
(14.97)\end{array}$ & $\begin{array}{l}43 \\
(46,24 \%)\end{array}$ & $\begin{array}{l}54 \\
(58,06 \%)\end{array}$ \\
\hline
\end{tabular}

Table 2.- Performance of ACR 1999 and ACR/EULAR preliminary Criteria for GPA in Chilean Patients

\begin{tabular}{|l|l|l|}
\hline GPA Clasification Criteria & ACR 1990 & $\begin{array}{l}\text { ACR/EULAR 2017, } \\
\text { preliminary Criteria }\end{array}$ \\
\hline Sensitivity & $57,6 \%$ & $91,5 \%$ \\
\hline Especificity & $70,6 \%$ & $91,2 \%$ \\
\hline Positive Predictive Value & $77,3 \%$ & $94 \%$ \\
\hline Negative Predictive Value & $49 \%$ & $86 \%$ \\
\hline Positive Likelihood Ratio & 1,96 & 10,3 \\
\hline Negative Likelihood Ratio & 0,6 & 0,9 \\
\hline accuracy & 0,62 & 0,91 \\
\hline
\end{tabular}

Conclusions: In our population, provisional 2017 ACR/EULAR criteria for classificatión of GPA have better acurracy than ACR 1990 classification criteria.

References:

[1] -Leavitt RY, Fauci AS, Bloch DA, Michel BA, Hunder GG, Arend WP, Calabrese LH, Fries JF, Lie JT, Lightfoot RW Jr, et al.The American College of Rheumatology 1990 criteria for the classification of Wegener's Granulomatosis. Arthritis Rheum 1990;33:1101-7.

[2] -Luqmani, R, Merkel, P. New Clasification Criteria for ANCA-Associated Vasculitis: Implication for Clinical Practice. Lecture, 2016 ACR/ARHP Annual Metting.

Disclosure of Interest: None declared

DOI: 10.1136/annrheumdis-2017-eular.6732

\section{AB0591 EFFICACY AND SAFETY PROFILE OF INTRAVENOUS CYCLOPHOSPHAMIDE TREATMENT IN ELDERLY PATIENTS WITH SYSTEMIC VASCULITIS}

S. Shimoyama ${ }^{1}$, T. Kurita ${ }^{1}$, S. Fukaya ${ }^{2}$, M. Kanda ${ }^{2}$ on behalf of Japanese Red Cross Kitami Hospital, Hokkaido P.W.F.A.C. Obihiro Kosei Hospital. ${ }^{1}$ Internal medicine/ General medicine, Japanese Red Cross Kitami Hospital, Kitami; ${ }^{2} 3 r d$ Department of Internal medicine, Hokkaido P.W.F.A.C. Obihiro-Kosei General Hospital, Obihiro, Japan

Background: Intravenous cyclophosphamide is mainstay of remission induction and dose reduction of glucocorticoid in patients with systemic vasculitis. However, little evidence has yet shown the safety profile of intravenous cyclophosphamide, especially in elderly patients.

Objectives: To evaluate efficacy and safety of patients diagnosed as systemic vasculitis and treated with intravenous cyclophosphamide.

Methods: This retrospective study comprised the patients with active systemic vasculitis who were admitted to Kitami Red Cross Hospital and Obihiro-Kosei General Hospital from April 2009 to March 2016. These patients were treated with intravenous cyclophosphamide plus conventional therapy (IVCY group) or only with conventional therapy (glucocorticoid/azathioprine/tacrolimus and methotrexate) (conventional therapy group). The patients treated with oral cyclophosphamide or rituximab were excluded. Primary endpoint was defined as death or serious infections. Prognostic factors in IVCY group were analyzed by multivariate Cox regression methods.

Results: This study comprised 90 patients with active systemic vasculitis (61 microscopic polyangiitis, 9 eosinophilic granulomatosis with polyangiitis, 10 granulomatosis with polyangiitis, and 10 polyarteritis nodosa). Fifty-one patients were over 70-year-old (26 patients in IVCY group). The mean observation period was 30.1 months. IVCY group had a trend for higher event-free survival rate as compared with conventional therapy group $(p=0.19)$. IVCY group had significantly higher cumulative survival rate as well $(\mathrm{p}=0.04)$. Age ( $\mathrm{HR}=1.07,95 \% \mathrm{Cl} 1.01-$ 1.14, $\mathrm{p}=0.03$ ), five factor score ( $F F S, H R=3.39,95 \% \mathrm{Cl} 1.36-8.44, \mathrm{p}=0.01)$ and glucocorticoid dose at 24 weeks (GC24, HR=1.14, 95\% Cl 1.05-1.22, $\mathrm{p}=0.001$ ) were identified as risk factors for events. In patients over 70-year-old, IVCY group had a trend for higher cumulative survival rate as well $(p=0.07$, Figure1). FFS $(\mathrm{HR}=3.30,95 \% \mathrm{Cl} 1.19-9.15, \mathrm{p}=0.02)$ and $\mathrm{GC} 24(\mathrm{HR}=1.10,95 \% \mathrm{Cl} 1.02-1.20$, $\mathrm{p}=0.01$ ) were identified as risk factors for events.

Table 1. Background of patients with IVCY group and conventional therapy group

\begin{tabular}{lccc}
\hline & IVCY group $(\mathrm{n}=54)$ & conventional therapy group $(\mathrm{n}=36)$ & P value \\
\hline MPA,n $(\%)$ & $36(67 \%)$ & $25(69 \%)$ & 0.95 \\
EGPA, $\mathrm{n}(\%)$ & $6(11 \%)$ & $3(8 \%)$ & 0.56 \\
GPA, $(\%)$ & $8(15 \%)$ & $2(6 \%)$ & 0.36 \\
PN,n $(\%)$ & $4(7 \%)$ & $6(17 \%)$ & 0.19 \\
age $(\mathrm{y})$ & $66.4 \pm 15.5$ & $72.3 \pm 15.9$ & 0.09 \\
Male, $n(\%)$ & $19(35 \%)$ & $13(36 \%)$ & 0.93 \\
FFS $(\mathrm{d}=0)$ & $0.6 \pm 0.7$ & $0.7 \pm 0.8$ & 0.40 \\
BVAS $(\mathrm{d}=0)$ & $10.9 \pm 5.5$ & $10.5 \pm 6.4$ & 0.71 \\
PSL $(\mathrm{mg}, \mathrm{d}=0)$ & $50 \pm 11$ & $38 \pm 13$ & 0.39 \\
PSL pulse, $\mathrm{n}(\%)$ & $18(33 \%)$ & $22(61 \%)$ & 0.01 \\
AZA,n $(\%)$ & $13(24 \%)$ & $14(39 \%)$ & 0.10 \\
MTX,n $(\%)$ & $2(4 \%)$ & $4(11 \%)$ & 0.17 \\
\hline
\end{tabular}

Abbreviation: MPA (microscopic polyangiitis), EGPA (eosinophilic granulomatosis with polyangiitis), GPA (granulomatosis with polyangiitis), PN (polyarteritis nodosa), BVAS (Birmingham Vasculitis Activity Score), AZA (azathioprine), MTX (methotrexate), $d=0$ means day 0 ; the start of the treatment.

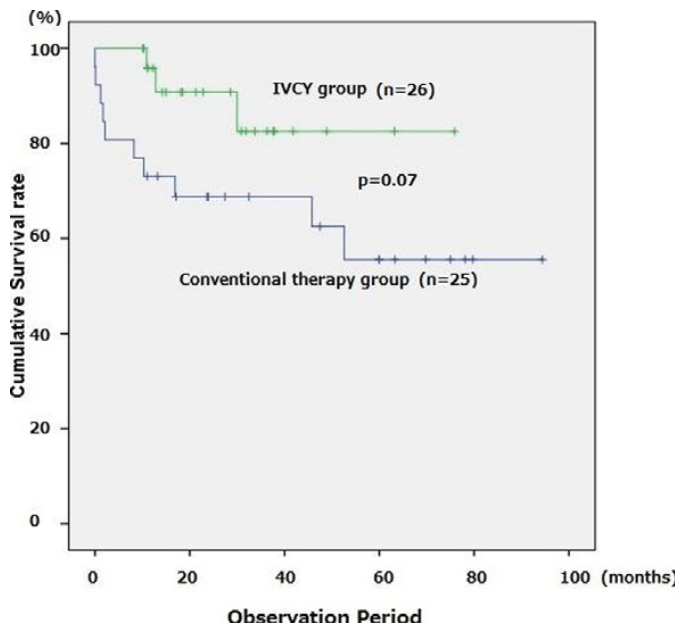

Conclusions: Intravenous cyclophosphamide treatment had acceptable safety profile even in elderly patients.

Disclosure of Interest: None declared

DOI: 10.1136/annrheumdis-2017-eular.3338

\section{AB0592 PULMONARY ARTERY ANEURYSM IN BEHÇET'S DISEASE: RETROSPECTIVE MONOCENTRIC TUNISIAN STUDY}

S. Hammami, A. Sondes, R. Klii, M. Kechida, M. Ben Nasr, I. Kochtali. Internal medicine, University Hospital F Bourguiba Monastir, Monastir, Tunisia

Background: Behcet's disease (BD) is a chronic inflammatory disorder. Arterial inflammatory involvement includes predominantly aortic and pulmonary aneurysmal lesions, affects about $10 \%$ of patients with BD. They account for the severity of the disease and are a leading cause of death

Objectives: To investigate the frequency of Behcet's disease with pulmonary artery aneurysm (PAA). We aimed to review PAA and other systemic involvements associated with PAA in $\mathrm{BD}$ and to provide a review of diagnostic techniques, treatment and prognosis

Methods: 243 BD patients were recruited for this study (152 men, 91 women, mean age $31.7 \pm 7$ years. Diagnosis of BD was made according to the international study group for Behçet's disease [International Study Group for Behçet's Disease, lancet 1990; 335: 1078-80]. All patients underwent full clinical examination, routine laboratory investigations. Chest X-rays and pulmonary CT angiography were performed on all patients with pulmonary involvement.

Results: Eight of the patients have pulmonary aneurysm, all of them are male, mean age $32.6 \pm 13$, The mean disease duration until PAA appear was $2.8 \pm 3.5$ years. The main pulmonary symptoms were as follows: dyspnea $87 \%$, cough $50 \%$, hemoptosis: $75 \%$, fever $37 \%$. Other systemic involvements associated PAA are as follows: buccal (100\%) and genital (75\%) ophthalmic $25 \%$, neurological $50 \%$, cardiac $25 \%$. 3 patients presented with Hughes Stovin syndrome. The treatment includes corticosteroids, colchicine and immunosuppressant agents (Cyclophosphamid or azathioprine), only two patients reveive coil embolization. At follow up for a median of 4 years ( 1 to 25 years), three patients died because they stopped their medication.

Conclusions: The prognosis of PAA is poorer than other lesions involved in $\mathrm{BD}$, treatment (immunosuppressant agents, colchicine) seems to improve the prognosis. It is important to maintain the immunosuppressive therapy and a regular follow-up to prevent these complications 